\title{
Psychiatry in Qatar
}

\author{
M. Fakhr El-Islam
}

Qatar is an Arab country occupying a peninsula in the middle of the Western coast of the Gulf separating Persia from Arabia. It is an oil-exporting country with a population of about $400000,70 \%$ of which live in the capital, Doha. The increase in wealth with the discovery of oil made it possible for many young members of extended families to move away and led to the emergence of nuclear families. In spite of this geographical separation, most nuclear families consider themselves extensions of their original extended families and maintain ties with them for the purpose of consultation, e.g. to arrange marriages, buffer disputes or mediate to secure benefits.

Non-Qataris usually come to Qatar for work on a temporary basis and the majority leave their spouse and children behind in their home countries. Immigration leads to a temporary state of interactional deprivation in the new environment and vulnerable persons may be thrown into mental ill-health. They have diffuse supportive social networks which are usually nationality-bound.

Illness concepts and illness behaviour vary according to the type of problem confronted. Somatic complaints call for medical help. For emotional and behavioural problems the help of a native healer (Motawwaa) is sought. Social problems cannot be dealt with as such and have to be disguised as somatic, emotional or behavioural problems.

Non-Qataris tend to present less often and later in the course of their illness (physical or psychiatric) than Qataris. The pressure is very high on immigrants to perform their jobs adequately and regularly. Having time off work to receive medical attention counts against them and so it is often late after the onset of their illness that they eventually present to the health service clinics. On the other hand, they are cooperative with treatment procedures and follow up more regularly than Qataris.

In the past, psychiatric disorder was masked by a number of factors. The cultural beliefs of Quataris attributed emotional and behavioural disorders to supernatural agents and the somatically orientated medical practitioners attributed somatic presentations of psychiatric patients to vague hypothesised physical disorders. Since the introduction of a specialist psychiatric service in 1971 more cases could be identified as psychiatric illnesses and, through professional contacts with the psychiatric department, general practitioners became better oriented to psychiatric problems.

Symptoms are concretely expressed according to the way they are perceived by Gataris. Tension is often described as body aches which are attributed to deep locations and even described as 'bone aches' because no superficial lesion is detectable. Abstract symptoms may be conceived concretely as if they were somatic, e.g. poor concentration on reading may be attributed by school children and their parents to their eyes 'not catching properly' and these children are often taken to the eye clinic. Worries about unpleasant anticipations are ascribed to the heart which is held to tell the patient about them. Eliciting the factors precipitating psychiatric disorder is often difficult. Many Qataris do not conceive the possible relationship between their symptoms and recent life events although the sequence of events and content of symptoms suggest such a relationship.

Qatar is one of the few middle-eastern countries which have never had a mental hospital. The first general hospital (Doha Hospital) built in 1948 started to accept psychiatric patients in 1956, when another general hospital (Rumaillah Hospital) was built. Patients were looked after by a general practitioner. Prior to their management in Doha Hospital, psychotic patients used to be restrained and fed at home or in prison according to their family status and resources.

The establishment of a psychiatric service in 1971 came shortly after the country's independence. At about the same time consultations and out-patient and in-patient treatments in all branches of medicine became unconditionally free of charge for patients from all nationalities. At present the psychiatric service consists of one psychiatric out-patient clinic, an in-patient unit, a 


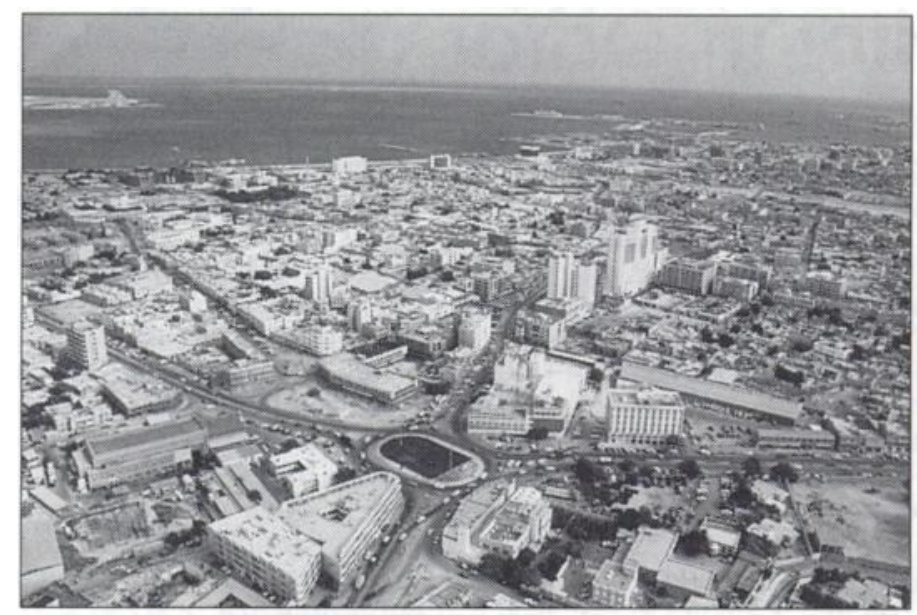

Figure 1. Scene from Qatar.

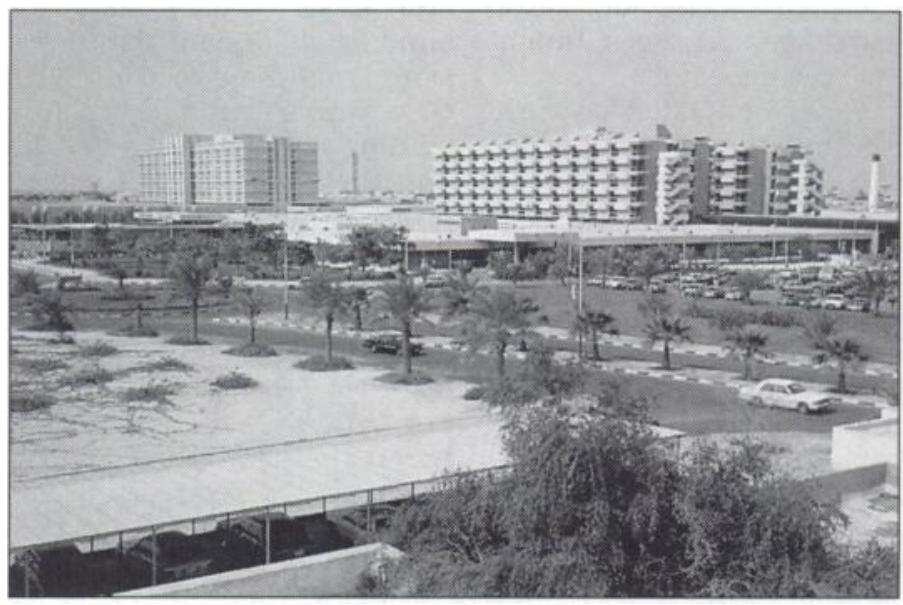

Figure 2. Hamad General Hospital, Doha-Qatar.

community nursing service and an emergency service. In 1982 a new medical complex known as the Hamad Medical Corporation, which includes the present department of psychiatry, was established.

The out-patient clinic started off with about 1200 consultations in 1971 but the number stabilised at about 14000 yearly over the past four years. The clinic offers a wide range of psychiatric treatments including psychopharmatherapeutic agents and various forms of cognitive, behavioural and supportive therapies at individual and family levels. No neurological cases are accepted at the clinic, and unless associated with psychiatric symptoms epileptics are not normally treated at the psychiatric clinic. The clinic therefore does not subscribe to the confusion in middleeastern countries where no distinction is made between neurology and psychiatry and where neurologists enjoy the lucrative field of psychiatric practice.

The concept of maintenance therapy is not entertained by many patients who abandon their treatment as soon as their symptoms abate. They often enquire as to which medicines act on which symptom and seem to presume that a given drug can only cope with 
one symptom. Qatari patients have a dependent attitude towards doctors. They are not keen on active participation in their own treatment or follow-up, are intolerant of side effects of tranquillisers and antidepressants, and do not do their 'homework' in relaxation therapy, training in assertion or behaviour therapy. Insight psychotherapy is too abstract and too dependent on patients' participation to be acceptable to many Qataris for whom talking cannot replace a prescription. However, supportive and behavioural psychotherapy and especially family therapy have been introduced and gained some acceptance, with the doctor perceived as the 'family friend'. The community nursing service was established in 1993. Nurses carry out domiciliary visits for assessment and home management of patients in liaison with family members. They give depot neuroleptic medication to psychotic patients, ensure compliance with out-patient follow-up appointments and help families bring patients to hospital when their condition makes this necessary.
The in-patient psychiatric unit has 56 beds: 40 for men and 16 for women. It provides recreational and occupational therapy in addition to the general methods of psychiatric treatment. Patients are admitted through the psychiatric out-patient clinic or the emergency service in the general hospital. All admissions are 'informal'. The family plays a major role in the decision to admit patients on the advice of the psychiatrist. Also discharge of patients from hospital and their extramural support and follow-up are the joint responsibility of the family and psychiatric team. The family in Qatar is still the best psychosocial agent. For expatriates not living in families a circle of friends often takes over this function. Mental health legislation is not required for the mental health services in Qatar.

M. Fakhr El-Islam, Senior Consultant Psychiatrist, Chairman, Department of Psychiatry, Hamad Medical Corporation, Doha-Qatar

\section{Management for Psychiatrists}

\section{Second Edition}

\section{Edited by Dinesh Bhugra and Alistair Burns}

Since the last edition rapid changes in the NHS have meant that clinicians have had even less time to manage change and keep up to date with health reforms. For this new edition, all the existing material has been extensively revised. In addition, eight new chapters have been added, including a section on changes and conflicts covering large areas of potential difficulty that clinicians may have to deal with.

As before, the emphasis is on how to get the best for and from services. Practical advice is given on management. Negotiation techniques and time and stress management are also covered.

$\bullet £ 20.00 \bullet 360 p p . \bullet 1995 \bullet$ ISBN 0902241850

Available from bookshops and from the Publications

Department, Royal College of Psychiatrists, 17 Belgrave Square,

London SW1X 8PG (Tel. 0171-235 2351 extension 146) 